\title{
Prediction of huge X-ray Faraday rotation at the Gd $\mathbf{N}_{4,5}$ threshold
}

\author{
J.E. Prieto, F. Heigl, O. Krupin, G. Kaindl and K. Starke \\ Institut für Experimentalphysik, Freie Universität Berlin, Arnimallee 14, D-14195 Berlin, Germany
}

(Dated: October 31, 2018)

\begin{abstract}
X-ray absorption spectra in a wide energy range around the $4 \mathrm{~d}-4 \mathrm{f}$ excitation threshold of $\mathrm{Gd}$ were recorded by total electron yield from in-plane magnetized Gd metal films. Matching the experimental spectra to tabulated absorption data reveals unprecedented short light absorption lengths down to $3 \mathrm{~nm}$. The associated real parts of the refractive index for circularly polarized light propagating parallel or antiparallel to the Gd magnetization, determined through the KramersKronig transformation, correspond to a magneto-optical Faraday rotation of $0.7^{\circ}$ per atomic layer. This finding shall allow the study of magnetic structure and magnetization dynamics of lanthanide elements in nanosize systems and dilute alloys.
\end{abstract}

PACS numbers: 75.30.-m, 75.70.-i, 78.20.Ls, 78.70.Dm

Resonance enhancements of X-ray magnetic scattering cross sections at inner-shell absorption edgest 1 h have been used for years to investigate the magnetic structure of lanthanide and actinidet systems in the hard X-ray regime (above $2 \mathrm{keV}$ ). The experimental demonstration of large changes in the specularly reflected X-ray intensity at the $\mathrm{Fe}_{2,3}$ edge $\mathrm{E}$ upon magnetization reversal initiated the ongoing search for magneto-optical (MO) ef-

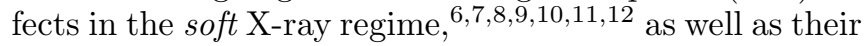
application to olement-specific studies of heteromagnetic systems $13,44,15,16,17]$

Yet, in analyzing soft X-ray MO signals from thin films and multilayer systems with thicknesses comparable to ray wavelength, previous investigations hare shown 13318 that a comparison with model calculations 19 of the reflected specular intensity (based on the Fresnel equations) is needed in order to extract a layerresolved sample magnetization profile. Several experimental determinations of soft X-ray MO constants have been reported 10.10 for ferromagnetic transition metals in the region of the $\mathrm{L}_{2,3}$ thresholds, but none so far for the lanthanide elements, despite their wide recognition as, e.g., constituents of exchange-spring magnets 20 and magnetic recording media21. Only recently has it been demonstrated that sizeable $\mathrm{MO}$ signals are obtained from lanthanide elements in the soft X-ray region at the $\mathrm{N}_{4,5}$ thresholds. 22

Here we show that calibrated $\mathrm{N}_{4,5}$ absorption spectra from magnetized Gd metal, recorded with circularly polarized (CP) light in the energy interval from 110 to $200 \mathrm{eV}$, yield an X-ray absorption coefficient up to three times larger than expected. The magnetizationdependent absorption of $\mathrm{CP}$ light at the $\mathrm{Gd}_{4,5}$ giant resonance maximum, described by the imaginary part of the refraction index, is accompanied by a huge change in light propagation speed upon magnetization reversal, a dispersive effect described by the real part of the refraction index, implying a Faraday rotation (FR) of about $0.7^{\circ}$ per atomic layer. Thus, even very small or diluted lanthanide systems are expected to show a measurable effect.

The absorption experiments were performed at the high-resolution UE56 undulator beamline 23 of the Berliner Elektronenspeicherring für Synchrotronstrahlung (BESSY II). The photon energy resolution was set to about $100 \mathrm{meV}$ (FWHM) which is well below the intrinsic width of the narrow $\mathrm{Gd} \mathrm{N}_{4,5}$ pre-edge absorption lines.24 The photon energy interval from 110 to $200 \mathrm{eV}$ was scanned at slow speed by a synchronized movement of monochromator and undulator. This synchronization is essential to properly normalize the absorption spectra and allows one to exploit the high flux of the undulator beamline of about $10^{14}$ photons $/(\mathrm{s} \cdot 100 \mathrm{~mA} \cdot 0.1 \%$ bandwidth $)$ over a wide energy range. The degree of circular polarization at this Sasaki-type undulator beamline is practically $100 \% .23$

The absorption spectra were recorded in total-electron yield (TEY) mode using a high-current channeltron. To suppress the background of secondary electrons from the chamber walls, both the sample and a retarding grid placed in front of the channeltron, were biased with a low-voltage battery. For signal stability, high voltage was supplied to the channeltron cathode by a $3.2 \mathrm{kV}$ battery box. The electron-yield current was amplified by an electrometer (set to $3 \mathrm{~ms}$ integration time for a scan speed of typically $0.1 \mathrm{eV}$ per second). We used a light incidence angle of $30^{\circ}$ with respect to the film plane, in order to compromise between a large projection of the $\mathrm{CP}$ light wave-vector onto the in-plane film-magnetization and the desired small sample reflectivity 25

Epitaxial Gd metal films of $(10 \pm 1) \mathrm{nm}$ thickness were prepared in situ by vapor deposition in ultra-high vacuum $\left(3 \times 10^{-11}\right.$ mbar base pressure; about $4 \times 10^{-10}$ mbar during deposition) on a $\mathrm{W}(110)$ singlecrystal substrate (for details of film preparation, see Ref. 26). For remanent in-plane sample magnetization, an external field was applied along [110] of the substrate, i.e., parallel to the easy magnetization axis of the Gd film, using a rotatable electromagnet 27 A compact visiblelight MO Kerr-effect setup 28 was used to routinely check the state of remanent magnetization of the Gd films, revealing square-shaped hysteresis loops with about $100 \mathrm{Oe}$ coercivity.

Figure 11 displays experimental absorption spectra in 


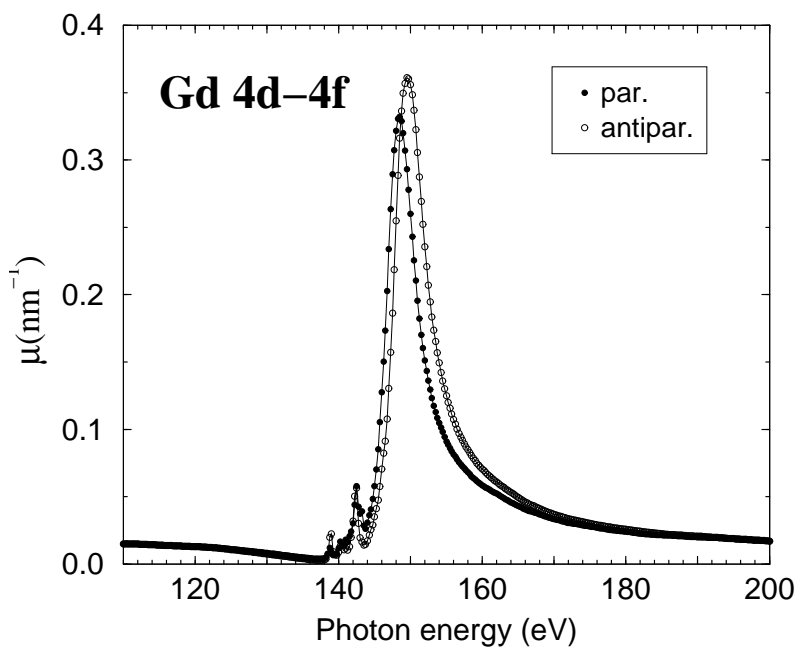

FIG. 1: Gd $\mathrm{N}_{4,5}$ absorption spectra from remanently magnetized $\mathrm{Gd}$ films at $T=30 \mathrm{~K}$. CP light was incident at $30^{\circ}$ with respect to the film plane, i.e. mainly parallel (filled symbols) and antiparallel (open symbols) to the in-plane sample magnetization.

the region of the Gd $\mathrm{N}_{\text {threshold. The spectra were }}$ corrected for saturation 29.30 assuming 0.3 for the ratio of electron escape depth to minimal X-ray absorption length. The photon energy range of the present spectra is significaptly wider than of those measured in previous studies 24 B1, including the wide asymmetric flanks of the $4 \mathrm{~d} \rightarrow 4 \mathrm{f}$ giant resonance (Beutler-Fano profile). This allows one to calibrate the absorption spectra by match ing both ends to the tabulated absorption coefficient 32 at photon energies where the influence of the giant resonance is expected to be negligible. To this end we fixed the absorption coefficients $\mu_{ \pm}$at the low-energy $(110 \mathrm{eV})$ and high-energy sides $(200 \mathrm{eV})$ of the measured spectra to the values given by the tables of Henke et al 32 , 15.0 and $17.1 \times 10^{-3} \mathrm{~nm}^{-1}$ at 110 and $200 \mathrm{eV}$, respectively. This then defines the given scale of the ordinate in Fig. 1. In this way, the absoption coefficient is obtained with an error bar of $\pm 15 \%$ at the maximum, estimated from our experimental precision of $\pm 1 \%$ at both ends of the photon energy range, where the matching to the tabulated data was performed.

We note that the spectra in Fig. 1 1 show the same qualitative energy dependence as given earlier 24 , yet the previous spectra were scaled to the same maximum value for both magnetization directions without any correction for saturation effects.

The comprehensive X-ray data tables of Henke et al. 32 contain the lanthanide $\mathrm{N}_{4,5}$ absorption spectra by Zimkina et al 33 , who measured relative linear X-ray absorption lengths of nonmagnetized lanthanide samples To obtain an absolute apsorption length, Henke et al.32 followed Richter et al.34 who calibrated their gas-phase photoexcitation data by matching them to calculated cross sections. In this way they arrived at a maxi-
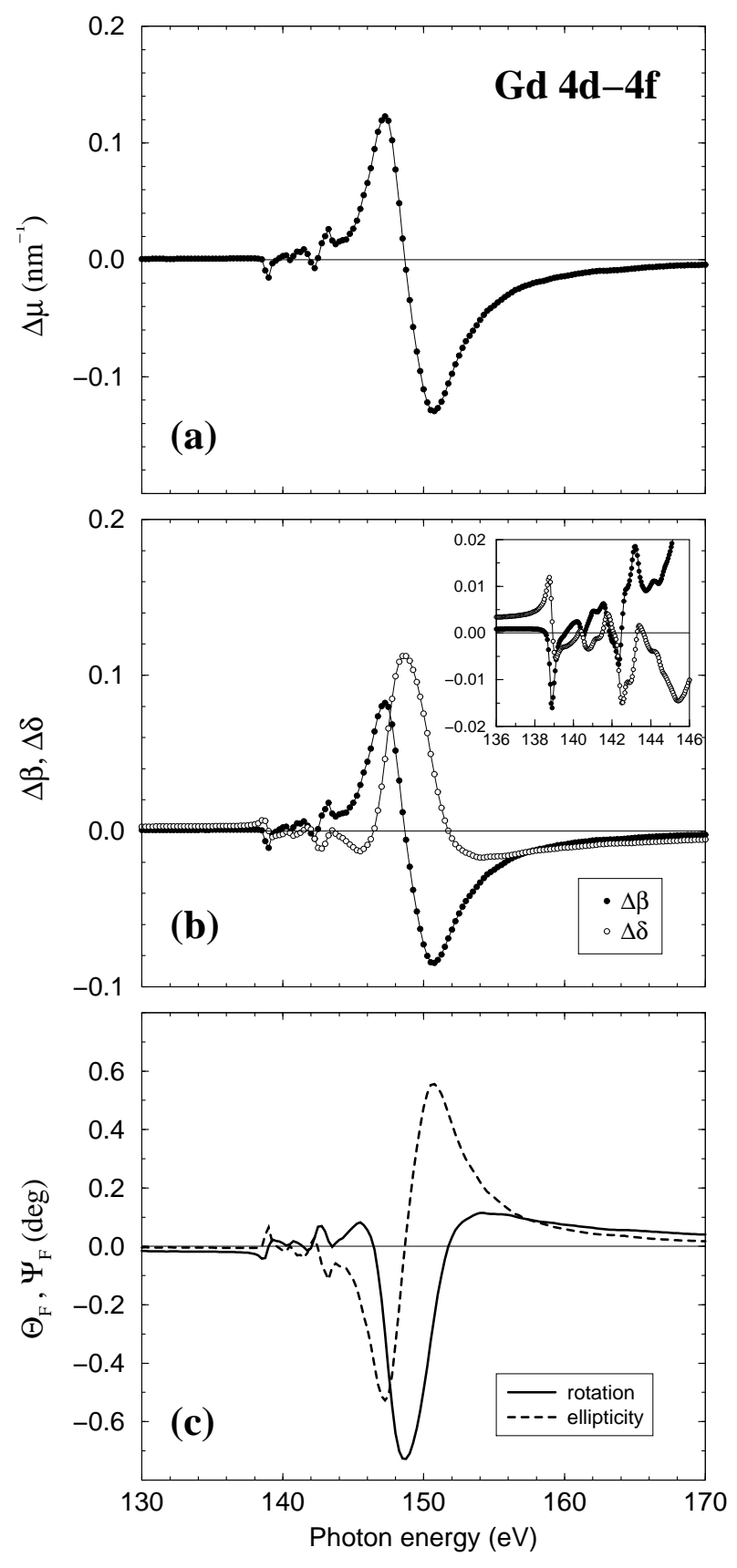

FIG. 2: (a) Difference spectrum, $\Delta \mu(\omega) \equiv\left(\mu_{+}(\omega)-\right.$ $\left.\mu_{-}(\omega)\right) / \cos \left(30^{\circ}\right)$, of the absorption spectra for opposite magnetizations given in Fig. 1. (b) Difference $\Delta \beta$ of the imaginary parts (filled symbols) and the associated difference $\Delta \delta$ of the real parts (open symbols) obtained through a Kramers-Kronig transformation. Inset: pre-edge range, measured $(\Delta \beta$, filled symbols) and calculated ( $\Delta \delta$, open symbols) with higher point density. (c) FR and ellipticity spectra calculated for linearly polarized light transmitted normally through a $0.3 \mathrm{~nm}$ thick Gd metal film magnetized perpendicular to the film plane. 
mum absorption coefficient at the nonmagnetic $\mathrm{Gd} \mathrm{N}_{4,5}$ peak of $\mu \approx 0.1 \mathrm{~nm}^{-1}$, corresponding to a linear X-ray absorption length $\lambda=1 / \mu \approx 10 \mathrm{~nm} .22$ By contrast, the calibrated experimental spectra from magnetized Gd in Fig. 1 reveal maximum values for the absorption coefficient of $0.33 \mathrm{~nm}^{-1}$ and $0.36 \mathrm{~nm}^{-1}$, for nearly parallel and antiparallel orientation of sample magnetization and photon spin, respectively. The corresponding linear absorption lengths are $3.0 \mathrm{~nm}$ and $2.8 \mathrm{~nm}$, with the quoted error of $\$ 1.5 \%$; they are about three times shorter than expected. 32 These soft X-ray absorption lengths are remarkably short, even compared with visible-light absorption lengths in metals of typically some $20 \mathrm{~nm}$.

The difference spectrum $\Delta \mu(\omega) \equiv\left(\mu_{+}(\omega)-\mu_{-}(\omega)\right) /$ $\cos \left(30^{\circ}\right)$, obtained from the experimental absorption spectra $\mu_{ \pm}(\omega)$, is displayed in Fig. 2a. The factor $1 / \cos \left(30^{\circ}\right)$ accounts for the finite experimental angle between the direcions of light propagation and magnetization. Apart from minor contributions from the weaker pre-edge transitions (inset of Fig. $2 \mathrm{~b}$ ) around $140 \mathrm{eV}$, the $\Delta \mu(\omega)$ spectrum exhibits an S-shape behavior, with a zero crossing near $149 \mathrm{eV}$. It originates from the very intense $4 \mathrm{~d}^{10} 4 \mathrm{f}^{7}\left[{ }^{8} \mathrm{~S}\right] \rightarrow 4 \mathrm{~d}^{9} 4 \mathrm{f}^{8}\left[{ }^{8} \mathrm{P}\right]$ transitions (dipole-allowed in LS coupling). For parallel orientation of photon spin and sample magnetization $\left(\Delta \mathrm{M}=+1\right.$ transitions), the intermediate ${ }^{8} P_{5 / 2}$ state at around $148 \mathrm{eV}$ is preferentially populated; for antiparallel orientation ( $\Delta \mathrm{M}=-1$ transitions), by contrast, the only allowed excitation is into the higher ${ }^{8} \mathrm{P}_{9 / 2}$ state at around $150 \mathrm{eV} 24$ The large difference in absorption coefficient for opposite magnetization directions (Fig. 2a) corresponds to a difference in the absorptive part $\Delta \beta \equiv \beta_{+}-\beta_{-}=\Delta \mu \lambda /(4 \pi)$ of the refractive index $n_{ \pm}=1-\delta_{ \pm}-i \beta_{ \pm}$. As shown in Fig. $2 \mathrm{~b}, \Delta \beta$ changes from +0.082 to -0.085 within $3.5 \mathrm{eV}$.

From the present data, the associated difference in the real part $\Delta \delta \equiv \delta_{+}-\delta_{-}$, for CP light propagating (exactly) parallel or antiparallel to the Gd magnetization, was derived using the Kramers-Kronig (KK) transformation for magnetic systems 35 the result is given in Fig. 20 $\mathrm{b}$ by open symbols. The accuracy of this integral transformation depends mainly on the spectral range available for integration. Within the extended photon energy range of $110-200 \mathrm{eV}$, the absorption spectra recorded for opposite magnetization directions appear to become asymptotically equal at both ends of the experimental photon-energy range (see Fig. [1). Hence the difference $\Delta \beta$ vanishes at the two boundaries, so that the result of the KK transformation is not affected by the choice of the experimental photon energy range. $\Delta \delta$ peaks right at the zero crossing of the absorptive part, where it amounts to $\Delta \delta \approx 0.11$ (see Fig. 2 $\mathrm{b}$ ).

For future applications, we use the experimental difference in the absorptive part, $\Delta \beta$, together with the calculated phase difference, $\Delta \delta$, to calculate the complex FR of Gd metal. Here we assume fully oriented $4 \mathrm{f}$ magnetic moments as existing, e.g., in the ferromagnetic phase at low temperatures $\left(T / T_{C} \ll 1\right)$. Real and imagi- nary parts of the $\mathrm{FR}, \Theta_{F}$ and $\Psi_{F}$, respectively, are given by the expressions 36

$$
\begin{aligned}
\tan \left(2 \Theta_{F}\right) & =2 \operatorname{Re}[a] /\left(1-|a|^{2}\right), \\
\sin \left(2 \Psi_{F}\right) & =2 \operatorname{Im}[a] /\left(1+|a|^{2}\right),
\end{aligned}
$$

where $d$ is the film thickness, $a=\tan (\Delta n \omega d / c)$ and $\Delta n=\left(n_{+}-n_{-}\right) / 2$. The $\Theta_{F}(\omega)$ and $\Psi_{F}(\omega)$ spectra of Gd metal at the $\mathrm{N}_{4,5}$ threshold are presented in Fig. 2 $c$ for linearly polarized (LP) light transmitted in normal direction through a $0.3 \mathrm{~nm}$ (1 monolayer) thick Gd metal film magnetized perpendicular to the film plane, either parallel or antiparallel to the light propagation direction. The spectra predict a $\mathrm{FR}$ of $\Theta_{F}=(0.73 \pm 0.11)^{\circ}$ per $0.3 \mathrm{~nm}$ $\left((2.4 \pm 0.4)^{\circ} / \mathrm{nm}\right)$ near $149 \mathrm{eV}$, right at the zero crossing of the absorption difference for CP light in Fig. 2a. At this photon energy, $\Theta_{F}$ is accompanied by a vanishing Faraday ellipticity $\Psi_{F}$ (cf. Fig. 2 2 ). To our knowledge this is by far the largest specific FR reported. It is 9 times larger than the specific rotation maximum at the $\mathrm{Fe}_{3}$ threshold 10 and some 70 times (50 times) larger than in the visible (infrared) region of Fe metal.

With the predicted specific FR at $\mathrm{N}_{4,5}$ thresholds, already some $10^{15}$ lanthanide atoms per $\mathrm{cm}^{2}$ as in, e.g., a single atomic layer, a very dilute film, or nanosize particles, should be sufficient to show a measurable rotation. Note that it is not at all evident that continuum classical electrodynamics, as used in this work for the $10 \mathrm{~nm}$ thick Gd metal films, will still be appropriate when approaching atomic dimensions.

The huge $\mathrm{FR}$ at $\mathrm{Gd} \mathrm{N} \mathrm{N}_{4,5}$ is due to the very large electric dipole (E1) transition probability of $4 \mathrm{~d}^{10} 4 \mathrm{f}^{\mathrm{n}} \rightarrow 4 \mathrm{~d}^{9} 4 \mathrm{f}^{\mathrm{n}+1}$ transitions ( $n=7$ for Gd). Hence, when applying magnetized Gd films as a method to rotate the plane of light polarization at the fixed photon energy of $149 \mathrm{eV}$, the strong absorption at the $\mathrm{Gd} \mathrm{N}_{4,5}$ maximum (cf. Fig. 1) leads to a very short penetration length of the order of only $3 \mathrm{~nm}$. In order to obtain, e.g., a FR of $\pm 45^{\circ}$ for opposite film magnetizations, one would use a $18.5 \mathrm{~nm}$ thick Gd film, with an inevitable intensity reduction by a factor of $4.5 \times 10^{2}$. Despite this substantial loss in intensity, which leads to a transmitted flux of about $10^{11}$ photons $/(\mathrm{s} \cdot 100 \mathrm{~mA} \cdot 0.1 \%$ bandwidth $)$ at a typical third-generation undulator beamline, Gd films might well be useful in differential (lock-in technique) experiments, where fast switching of the X-ray polarization plane (ns time scale) is required. One could extend the photon energy range of this method to about $180 \mathrm{eV} 32$ by using heavier lanthanide elements.

\section{Acknowledgments}

J.E.P. thanks the Alexander-von-Humboldt Stiftung for generous support. We gratefully achnowledge the experimental help of Fred Senf and Rolf Follath (BESSY), and useful discussions with Jeff Kortright and Eric Gullikson (LBNL). This work was financed by the German 
1 D. Gibbs, D. R. Harshman, E. D. Isaacs, D. B. McWhan, D. Mills, and C. Vettier, Phys. Rev. Lett. 61, 1241 (1988), who discovered x-ray resonant magnetic scattering at the Ho $L_{3}$ absorption threshold.

2 E. D. Isaacs, D. B. McWhan, C. Peters, G. E. Ice, D. P. Siddons, J. B. Hastings, C. Vettier, and O. Vogt, Phys. Rev. Lett. 62, 1671 (1989).

3 D. F. McMorrow, D. Gibbs, and J. Bohr, in Handbook of Physics and Chemistry of Rare Earths, edited by K. A. Gschneidner, Jr. and L. Eyring (Elsevier, Amsterdam, 1999), vol. 26, p. 1.

4 D. Mannix, S. Langridge, G. H. Lander, J. Rebizant, M. J. Longfield, W. G. Stirling, W. J. Nuttall, S. Coburn, S. Wasserman, and L. Soderholm, Physica B 262, 125 (1999).

5 C.-C. Kao, C. T. Chen, E. D. Johnson, J. B. Hastings, H. J. Lin, G. H. Ho, G. Meigs, J. M. Brot, S. L. Hulbert, Y. U. Idzerda, and C. Vettier, Phys. Rev. B 50, 9599 (1994).

6 J. M. Tonnerre, L. Sève, D. Raoux, G. Soullié, B. Rodmacq, and P. Wolfers, Phys. Rev. Lett. 75, 740 (1995).

7 F. U. Hillebrecht, T. Kinoshita, D. Spanke, J. Dresselhaus, C. Roth, H. B. Rose, and E. Kisker, Phys. Rev. Lett. 75, 2224 (1995).

8 V. Chakarian, Y. U. Idzerda, and C. T. Chen, Phys. Rev. B 57, 5312 (1998).

9 M. Sacchi, C. F. Hague, L. Pasquali, A. Mirone, J.-M. Mariot, P. Isberg, E. M. Gullikson, and J. H. Underwood, Phys. Rev. Lett. 81, 1521 (1998).

10 J. B. Kortright and S.-K. Kim, Phys. Rev. B 62, 12216 (2000).

11 C. Schüßler-Langeheine, E. Weschke, A. Y. Grigoriev, H. Ott, R. Meier, D. V. Vyalikh, C. Mazumdar, C. Sutter, D. Abernathy, G. Grübel, and G. Kaindl, J. Electron Spectrosc. Relat. Phenomen. 114-116, 953 (2001).

12 H.-C. Mertins, P. M. Oppeneer, J. Kuneš, A. Gaupp, D. Abramsohn, and F. Schäfers, Phys. Rev. Lett. 87, 47401 (2001).

13 J. M. Tonnerre, L. Seve, A. Barbara-Dechelette, F. Bartolome, D. Raoux, V. Chakarian, C. C. Kao, H. Fischer, S. Andrieu, and O. Fruchart, J. Appl. Phys. 83, 6293 (1998).

14 Y. U. Idzerda, V. Chakarian, and J. W. Freeland, Phys. Rev. Lett. 82, 1562 (1999).

15 N. Weber, C. Bethke, and F. U. Hillebrecht, J. Appl. Phys. 85, 4946 (1999).

16 J. B. Kortright, S.-K. Kim, and H. Ohldag, Phys. Rev. B 61, 64 (2000).

17 O. Hellwig, J. B. Kortright, K. Takano, and E. E. Fullerton, Phys. Rev. B 62, 11694 (2000).

18 J. Geissler, E. Goering, M. Justen, F. Weigand, G. Schütz,
J. Langer, D. Schmitz, H. Maletta, and R. Mattheis, Phys. Rev. B 65, 020405 (2001).

19 S. A. Stepanov and S. K. Sinha, Phys. Rev. B 61, 15302 (2000).

20 E. E. Fullerton, J. S. Jiang, C. H. Sowers, J. E. Pearson, and S. D. Bader, Appl. Phys. Lett. 72, 380 (1998).

21 Y. Nakamura, J. Magn. Magn. Mater. 200, 634 (1999).

${ }^{22}$ K. Starke, F. Heigl, A. Vollmer, M. Weiss, G. Reichardt, and G. Kaindl, Phys. Rev. Lett. 86, 3415 (2001).

23 M. R. Weiss, R. Follath, K. J. S. Sawhney, F. Senf, J. Bahrdt, W. Frentrup, A. Gaupp, S. Sasaki, M. Scheer, H.-C. Mertins, D. Abramsohn, F. Schäfers, W. Kuch, and W. Mahler, Nucl. Instrum. Meth. Phys. Res. A 467, 449 (2001).

${ }^{24}$ K. Starke, E. Navas, E. Arenholz, Z. Hu, L. Baumgarten, G. van der Laan, C.-T. Chen, and G. Kaindl, Phys. Rev. B 55, 2672 (1997).

25 D. Alders, T. Hibma, G. A. Sawatzky, K. C. Cheung, G. E. van Dorssen, M. D. Roper, H. A. Padmore, G. van der Laan, J. Vogel, and M. Sacchi, J. Appl. Phys. 82, 3120 (1997).

26 K. Starke, Magnetic Dichroism in Core-Level Photoemission (Springer, Berlin, 2000).

27 F. Heigl, O. Krupin, G. Kaindl, and K. Starke, Rev. Sci. Instrum. 73, 369 (2002).

28 K. Starke, K. Ertl, and V. Dose, Phys. Rev. B 46, 9709 (1992).

29 G. van der Laan and B. T. Thole, J. Electron Spectrosc. Relat. Phenomen. 46, 123 (1988).

30 R. Nakajima, J. Stöhr, and Y. U. Idzerda, Phys. Rev. B 59, 6421 (1999).

31 S. Muto, S.-Y. Park, S. Imada, K. Yamaguchi, Y. Kagoshima, and T. Miyhara, J. Phys. Soc. Jpn. 63, 1179 (1994).

32 B. L. Henke, E. M. Gullikson, and J. C. Davis, At. Data Nucl. Data Tables 54, 180 (1993), www-cxro. lbl. gov/optical_constants.

33 T. M. Zimkina, V. A. Fomichev, S. A. Gribivskii, and I. I. Zhukova, Fiz. Tverd. Tela (Leningrad) 9, 1147 (1967), [Sov. Phys. Solid State 9, 1128 (1967)].

34 M. Richter, M. Meyer, M. Pahler, T. Prescher, E. v. Raven, B. Sonntag, and H.-E. Wetzel, Phys. Rev. A 40, 7007 (1989).

35 K.-E. Peiponen, E. M. Vartiainen, and T. Asakura, Dispersion, Complex Analysis and Optical Spectroscopy (Springer, Berlin, 1999).

36 A. K. Zvezdin and V. A. Kotov, Modern Magnetooptics and Magnetooptical Materials (Institute of Physics Publishing, Bristol, 1997). 